\title{
Heart rate variability and plasma biomarkers in patients with type 1 diabetes mellitus: Effect of a bout of aerobic exercise
}

\author{
Chadi P. Anaruma ${ }^{a}$, Maycon Ferreira Jr. ${ }^{a}$, Carlos H.G. Sponton ${ }^{a}$, \\ Maria A. Delbin ${ }^{b}$, Angelina Zanesco ${ }^{a,{ }^{*}}$ \\ ${ }^{a}$ Department of Physical Education, Institute of Biosciences, UNESP - Univ Estadual Paulista, Rio Claro, SP, Brazil \\ ${ }^{\mathrm{b}}$ Department of Structural and Functional Biology, Institute of Biology, UNICAMP - University of Campinas, \\ Campinas, SP, Brazil
}

\section{A R T I C L E I N F O}

\section{Article history:}

Received 27 July 2015

Received in revised form

24 September 2015

Accepted 20 October 2015

Available online 26 November 2015

Keywords:

Type 1 diabetes mellitus

Blood glucose

$\mathrm{NO}_{x}$

Thiobarbituric acid reactive

substances (TBARS)

Acute aerobic exercise

Heart rate variability

\begin{abstract}
A B S T R A C T
Objective: The aim of this study was to evaluate: (1) the cardiovascular parameters and plasma biomarkers in people with type 1 diabetes mellitus (T1DM) at baseline; and (2) the heart rate variability (HRV) and blood glucose in response to a session of aerobic exercise $(\mathrm{AE})$ and during recovery period.

Research design and methods: Adults (18-35 years) were divided into two groups: control (CT, $n=10)$ and T1DM $(n=9)$. Anthropometric, cardiovascular, and biochemical parameters, and aerobic capacity (indirect peak oxygen uptake, $\mathrm{VO}_{2 \text { peak }}$ ) were evaluated at baseline. Thirty minutes of AE (40-60\% intensity) was performed on a treadmill. Blood glucose and HRV were determined at rest, during $\mathrm{AE}$, and during the recovery period.

Results: Anthropometric measurements, cardiovascular parameters, aerobic capacity, and biochemical parameters were similar between the groups at baseline. In the T1DM group, blood glucose, glycated hemoglobin, and thiobarbituric acid reactive substances concentrations were increased while nitrite/nitrate $\left(\mathrm{NO}_{x}{ }^{-}\right)$levels were reduced. During $\mathrm{AE}$, the magnitude of the reduction of blood glucose was greater than that during the recovery period in the T1DM group. The RR intervals and SDNN were reduced at rest as well as in the recovery period in T1DM subjects, whereas the RMSSD and pNN50 were only reduced during the recovery period. No changes were observed in low frequency (LF), high frequency (HF), and LF/HF ratio.

Conclusion: Our study shows that T1DM patients on insulin therapy have poor blood glucose control with greater lipid peroxidation and lower $\mathrm{NO}_{x}{ }^{-}$levels, accompanied by an imbalance in autonomic function detected by the challenge of $\mathrm{AE}$.
\end{abstract}

(C) 2015 Elsevier Ireland Ltd. All rights reserved.

\footnotetext{
* Corresponding author at: Institute of Biosciences, Av 24-A, 1515 - Bela Vista, Rio Claro, 13506-900 SP, Brazil. Tel.: +55 19 35264324 ; fax: +551935264320 .

E-mail address: azanesco@rc.unesp.br (A. Zanesco).

http://dx.doi.org/10.1016/j.diabres.2015.10.025

0168-8227/(C) 2015 Elsevier Ireland Ltd. All rights reserved.
} 


\section{Introduction}

Diabetes mellitus (DM) is a chronic disease clinically characterized by hyperglycemia which, in turn, can activate several signaling pathways such as protein kinase $C$, advanced glycation end-products (AGEs) and polyol leading to significant reactive species oxygen (ROS) production [1-4]. Furthermore, a number of studies have systematically shown that ROS overproduction and a decrease in nitric oxide (NO) bioavailability are the primary causes of most complications in patients with type 1 (T1DM) and type 2 DM (T2DM) [5]. Cardiovascular autonomic neuropathy (CAN) is considered a common complication in people with diabetes, causing abnormalities in heart rate control and vascular dysfunction and is considered the most important cause of mortality in T1DM [6,7]. However, the diagnosis of CAN is only detected when complications are already established and the burden and outcomes are, in general, irreversible. Thus, it is critical to identify any signs of CAN in the early stage of T1DM in an attempt to prevent its complications.

Heart rate variability (HRV) analysis has been largely used as an indirect indicator of autonomic nervous system function $[8,9]$. Increased sympathetic and/or decreased parasympathetic activities have been associated with high risk of sudden death. Indeed, several studies have shown that patients with T1DM and T2DM showed autonomic dysfunction [10-12]. Interestingly, most studies have focused on patients with T2DM and only a few studies have investigated T1DM and autonomic dysfunction.

In T1DM, hyperglycemia occurs as a consequence of impaired insulin production caused by pancreatic $\beta$-cell destruction; this occurs as a consequence of a complex process where genetic and environmental factors lead to an autoimmune response. At present, T1DM is not preventable and daily insulin administration is required as treatment; however, poor blood glucose control remains a challenge [13]. Thus, proper care and preventive actions to avoid CAN complications in these patients should be the highest priority in the healthcare system.

A healthy lifestyle is fundamental to the management of $\mathrm{DM}$ and the inclusion of aerobic exercise is advised to prevent the deleterious effects of the disease. Several studies have shown that regular physical exercise, of mild to moderate intensity, has cardiovascular benefits, and physically active patients have increased longevity associated with reduction in morbidity and mortality [14]. Therefore, this study had two objectives: (1) to examine cardiovascular parameters, redox state (concentration of thiobarbituric acid reactive substances (TBARS) and superoxide dismutase (SOD) activity), and concentrations of $\mathrm{N}^{\varepsilon}$-(carboxymethyl) lysine (CML) and nitrite/nitrate $\left(\mathrm{NO}_{x}{ }^{-}\right)$that reflect AGEs and nitric oxide formation, respectively, in patients with T1DM at baseline; and (2) to evaluate the HRV and concentrations of blood glucose and lactate in response to a challenge of aerobic exercise session as well as during the recovery period. The objective of this study was to detect early autonomic dysfunction in patients with diabetes without cardiovascular diseases that could be associated with plasma biomarkers, with a view to preventing complications of T1DM and encourage patients to improve metabolic control of blood glucose, as well as providing a feasible test for the physician to detect early autonomic dysfunction in this population.

\section{Methodology}

\subsection{Study participants}

The study was approved by the Ethical Committee of Institute of Bioscience at the Sao Paulo State University (protocol: 5128). All the volunteers were recruited through advertisements in Rio Claro-SP, BRA. Data collection was carried out from May 2012 to November 2013. A total of 19 male volunteers were eligible to participate in the present study. They were divided into two groups: control (CT, $n=10)$ and people with T1DM $(n=9)$. T1DM was determined according to previous medical diagnosis and in agreement with the International Diabetes Federation [15]. The inclusion criteria of this study were: males with T1DM (diagnosis of disease $<15$ years); age 18-35 years; body mass index $<30 \mathrm{~kg} / \mathrm{m}^{2}$; sedentary ( $<150$ min of moderate physical activity per week or $<60 \mathrm{~min}$ of vigorous physical activity per week); and serum creatinine $<2.0 \mathrm{mg} / \mathrm{dl}$. The exclusion criteria were: insulin resistance; arterial hypertension; congenital heart disease; use of $\beta$-adrenergic blocking agents and digoxin; renal dysfunction; respiratory, thyroid, and hematologic diseases; orthopedic disorders; smoking; alcohol abuse; and other conditions that preclude the practice of physical exercise. The inclusion criteria for the control group was similar, but without T1DM.

\subsection{Study protocol}

First appointment: The volunteers were informed about the procedures and risks of the study and signed a consent form in accordance with Ethical Committee of UNESP. The medical records, medication inventory, anthropometric parameters, resting heart rate (HR), systolic blood pressure (SBP), diastolic blood pressure (DBP), and glycemia were measured. Volunteers were familiarized with the treadmill (Movement RT $250 \mathrm{PRO})$ while $\mathrm{HR}$, blood glucose, and $\beta$-ketones were monitored.

Second appointment: Volunteers were familiarized with the treadmill while HR, blood glucose, and $\beta$-ketones were monitored.

Third appointment: To indirectly determine the peak oxygen uptake $\left(\mathrm{VO}_{2 \text { peak }}\right)$ the volunteers performed a 1 mile walk test as previously described [16] while HR, blood glucose, and $\beta$-ketone were monitored.

Fourth appointment: Baseline blood collection for biochemical analysis.

Fifth appointment: Acute aerobic exercise performance.

The experimental design of this cross-sectional study is illustrated in Fig. 1A.

\subsection{Acute exercise session}

All volunteers performed an exercise session in the afternoon, after 4:00 p.m. to avoid the interference of insulin administration (eight volunteers used insulin glargine and one volunteer 
A-Experimental Design

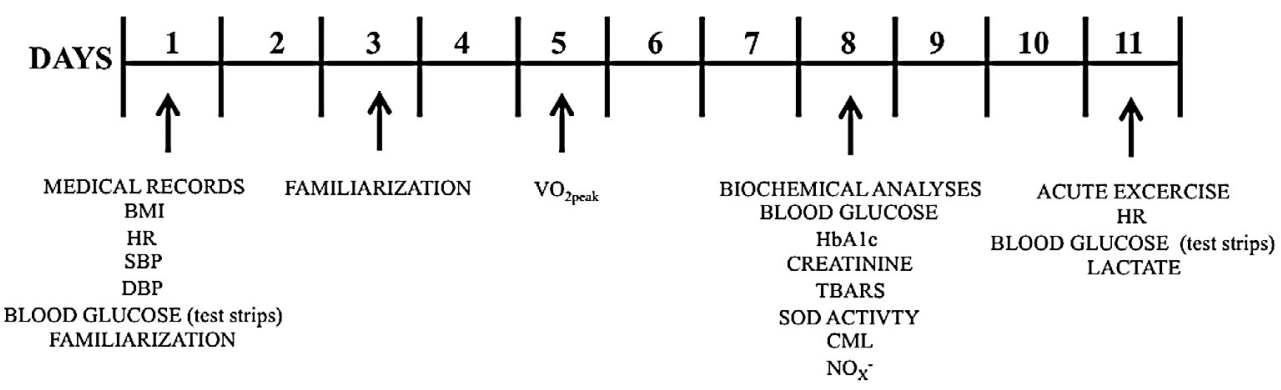

B-Acute Exercise Session

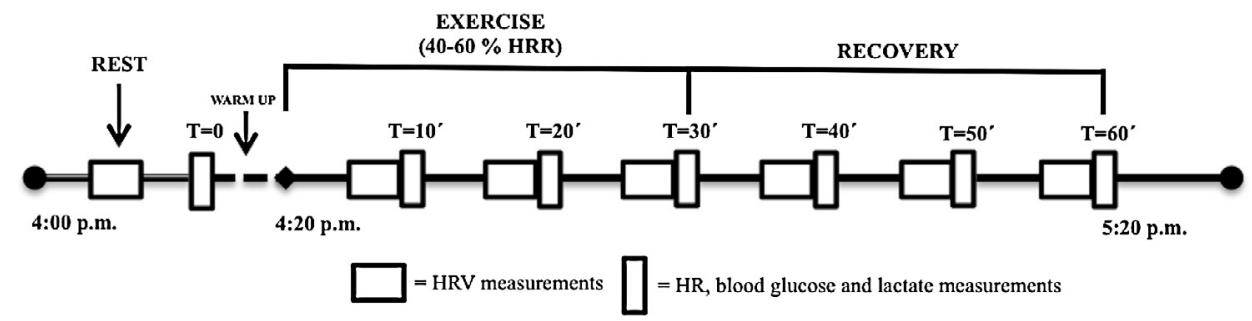

Fig. 1 - Experimental design and acute exercise session. BMI: body mass index; HR: heart rate; SBP: systolic blood pressure; DBP: diastolic blood pressure; $\mathrm{VO}_{2 \text { peak: }}$ peak oxygen consumption; HbA1c: glycated hemoglobin A1c; TBARS: thiobarbituric acid reactive substances; SOD ACTIVITY: superoxide dismutase activity; $\mathrm{CML}: \mathrm{N}^{\varepsilon}$-(carboxymethyl) lysine; $\mathrm{NO}_{x}^{-}:$nitrite/nitrate.

used Neutral Protamine Hagedorn insulin) during the morning (7:00 to 8:00 a.m.). The parameters were collected at rest $\left(T_{0}\right)$, during 10, 20, and 30 min of moderate exercise $\left(T_{10}, T_{20}\right.$, and $\mathrm{T}_{30}$, respectively), and during 10,20 , and $30 \mathrm{~min}$ of recovery period $\left(T_{40}, T_{50}\right.$, and $T_{60}$, respectively). The target zone of moderate exercise (40-60\% $\mathrm{HR}_{\text {reserve }}$ ) was determined according with Karvonen's formula [17]:

$\mathrm{HR}_{\text {reserve }}=\left[\left(\mathrm{HR}_{\max }-\mathrm{HR}_{\text {rest }}\right) \times \%\right.$ of target intensity $]+\mathrm{HR}_{\text {rest }}$ $\mathrm{HR}_{\max }=220-$ age $($ in years $)$

HR was monitored during all exercise sessions using a heart rate monitor (Polar RS8000CS, Polar, Finland). The speed was maintained constant at $5.5 \mathrm{~km} / \mathrm{h}$ and the grade was set according to the HR target zone.

During the exercise session, blood glucose was measured using standard test strips (Accu-Chek Performa Roche Diagnostics, Indianapolis-IN, USA). For lactate measurement, the blood samples were collected from the ear lobe and measured by enzymatic assay as previously described [18]. To avoid metabolic acidosis during the exercise session, we also monitored blood $\beta$-ketones using standard test strips (Optium Xceed Abbott Diabetes Care Inc., Alameda-CA, EUA). Fig. 1B illustrates the acute exercise session.

\subsection{Cardiovascular measurements}

\subsubsection{Blood pressure (BP) measurements}

After sitting for $15 \mathrm{~min}$, three consecutive BP measurements were made using semiautomatic equipment (Microlife MIBP3BTOA). Resting BP was calculated as the average of the measurements.

\subsubsection{Resting HR and heart rate recovery}

HR was measured using a heart rate monitor (Polar RS8000CS, Polar, Finland) after 15 min of rest in a seated position. At the end of the resting period HR values were obtained. Heart rate recovery was calculated as the reduction in heart rate at peak exercise to the rate $1 \mathrm{~min}$ after the cessation of exercise in both groups.

\subsection{Heart rate variability}

The measurement of heart rate variability using the $R R$ interval was performed for analysis of autonomic function as previously described [8]. The volunteers were instructed not to consume caffeine and/or alcohol, and to maintain their usual diet and not take exercise $48 \mathrm{~h}$ before the assessment. To collect the RR interval series and heart rate, volunteers remained at rest for $15 \mathrm{~min}$ in a seated position in an airconditioned and free ambient noise room using a beat-by-beat heart rate monitor (Polar RS800CX, Kempele, Finland). An interval of $5 \mathrm{~min}$ before exercise $\left(\mathrm{T}_{0}\right)$, during a bout of physical exercise $\left(T_{10-30}\right)$, and during recovery period $\left(T_{40-60}\right)$ for further analysis of the components in the time domain and the frequency domain were selected. The correction of artifacts and data processing were performed using the HRV Kubios 2.1 software (University of Kuopio, Finland). For the time domain, the mean SDNN and RMSSD components were analyzed, while for the frequency domain both low frequency (LF, 0:04-0:15 Hz) and high frequency (HF, 0:15-0:40 Hz) components were analyzed as well as the total power and LF/HF ratio by spectral analysis of the time-course of RR intervals using the Fast Fourier Transform (Welch's 
periodogram: $256 \mathrm{~s}$ window with $50 \%$ overlap). LF and HF components, which represent sympathetic and parasympathetic modulations, respectively, were expressed in milliseconds $\left(\mathrm{ms}^{2}\right)$.

\subsection{Blood collection and biochemical analyses}

Blood samples were collected after an $8 \mathrm{~h}$ overnight fast (between 7:00 and 8:00 a.m.) from antecubital vein using standard venipuncture methods. Samples were centrifuged (3000 rpm, $15 \mathrm{~min}$ ) and the supernatant (plasma and serum) were stored in aliquots at $-80^{\circ} \mathrm{C}$ for future analysis. The T1DM group was instructed to take their medications after blood collection.

Serum glucose, creatinine, and glycated hemoglobin (HbA1c) were determined using automated standard method (São Lucas Laboratory, Rio Claro-SP, BRA).

Plasma $\mathrm{NO}_{x}{ }^{-}$concentrations were measured using a commercially kit (Cayman Chemical, Ann Arbor-MI, USA). Briefly, plasma samples were ultrafiltrated through microfilter cups (Microcon Centrifugal Filter Units, $10 \mathrm{kDa}$; Millipore, Billerica-MA, USA). The $\mathrm{NO}_{x}{ }^{-}$concentration of the resulting filtrate was determined based on the enzymatic conversion of nitrate to nitrite by nitrate reductase. The absorbance was measured at $540 \mathrm{~nm}$ determined the nitrite concentration.

In order to analyze antioxidant status, plasma SOD activity was measured using commercially kit (Cayman Chemical, Ann Arbor-MI, USA). Briefly, SOD activity was assessed using a tetrazolium salt for detection of superoxide radicals generated by xanthine oxidase and hypoxanthine. One unit of SOD was defined as the amount of enzyme needed to Exhibit $50 \%$ dismutation of the superoxide radical. The assay provided the measurement of all three types of SOD and absorbance measured at $440 \mathrm{~nm}$.

The measurement of plasma TBARS is a well-established method for screening and monitoring lipid peroxidation and was measured using commercially available kit (Cayman Chemical, Ann Arbor-MI, USA). The malondialdehyde (MDA)2-thiobarbituric acid (TBA) adducts formed by the reaction of MDA and TBA under high temperature and acidic conditions were measured colorimetrically at $532 \mathrm{~nm}$.

Plasma CML (ng/mL) concentrations were measured using a commercial kit (Cell Biolabs Inc., San Diego-CA, USA). Briefly, plasma samples were used to determine the protein concentrations (Pierce BCA Protein Assay kit, Rockford-IL, USA) and samples were prepared in a concentration of $10 \mu \mathrm{g} / \mathrm{ml}$. After that, the CML protein adducts present in the sample were probed using an anti-CML antibody, followed by a horseradish peroxidase conjugated secondary antibody, and determined by comparing with standard curve absorbance measured at $450 \mathrm{~nm}$.

\subsection{Statistical analysis}

Data are expressed as mean \pm standard error mean. Unpaired Student's t-test was used to analyze the baseline values from CT versus T1DM groups. Repeated-measures one-way ANOVA followed by a Bonferroni's test was used to analyze the acute exercise results. The analyses were performed using GraphPad Prism Software (GraphPad Software, Prism 5, San
Diego-CA, USA). Values of $p<0.05$ were considered statistically significant.

\section{Results}

\subsection{Baseline measurements}

The characteristics of the study population are shown in Table 1. The anthropometric and cardiovascular parameters, as well as the aerobic capacity were similar between both groups. Despite insulin therapy, both blood glucose and HbA1c levels were significantly increased in T1DM group compared with CT subjects $(p<0.01$ and $p<0.0001$, respectively), approximately 100 and 75\%, respectively (Fig. 2, panels A and $B$, respectively). Concentrations of creatinine were significantly reduced in the T1DM group compared with CT subjects $(p<0.01)$, by approximately 19\% (Fig. 2C). Given that one of the hallmarks of diabetes state is associated oxidative stress, we examined the redox state in both groups. We found a marked increase in TBARS levels in the T1DM group of approximately $137 \%$ compared with CT ( $p<0.01)$, and without changes in SOD activity (Fig. 3, panels A and B, respectively). Furthermore, we also analyzed the concentrations of AGEs by measuring CML. Interestingly there was no difference in CML in either group (Fig. 3C).

Vascular dysfunction is highly present in patients with diabetes with an important endothelium dysfunction; thus, we also examined $\mathrm{NO}_{x}{ }^{-}$levels as an indirect measurement of NO production in both groups. We also found a marked decrease in $\mathrm{NO}_{x}{ }^{-}$levels in T1DM group (approximately 60\%) compared with CT subjects $(p<0.05)$ (Fig. 3D).

\subsection{Acute exercise}

\subsubsection{Blood glucose and lactate concentrations}

As expected, the T1DM subjects presented an increased blood glucose during the studied period compared with the CT group (Fig. 4A) $(p<0.01)$ whereas acute aerobic exercise was effective in lowering blood glucose in T1DM that was maintained during the recovery period (Fig. $4 \mathrm{~A})(p<0.001)$. Blood glucose concentration was only reduced in the CT group during the exercise period (Fig. 4A) $(p<0.001)$. As expected, the magnitude of blood

\begin{tabular}{lcc}
\multicolumn{3}{c}{ Table $\mathbf{1}$ - Characteristics of the study population. } \\
\multicolumn{2}{c}{ CT } & T1DM \\
\hline$n$ & 10 & 9 \\
Age (years) & $27 \pm 0.9$ & $24 \pm 1.7$ \\
Duration of diabetes (years) & - & $9.8 \pm 1.4(4-15)$ \\
Daily insulin (U/day) & - & $40 \pm 4.0$ \\
Weight $(\mathrm{kg})$ & $82.3 \pm 4.8$ & $76.4 \pm 4.6$ \\
BMI (kg/m $\left.{ }^{2}\right)$ & $26 \pm 1$ & $24 \pm 1.2$ \\
HR (bpm) & $62 \pm 1.8$ & $68 \pm 4.5$ \\
SBP (mmHg) & $113 \pm 1.8$ & $117 \pm 3.4$ \\
DBP (mmHg) & $68 \pm 1.5$ & $69 \pm 3.8$ \\
VO $_{2 \text { peak }}\left(\mathrm{mL} \mathrm{kg}\right.$ $\left.^{-1} \mathrm{~min}^{-1}\right)$ & $43.5 \pm 1.5$ & $46.0 \pm 1.2$
\end{tabular}

Data are mean \pm SEM. BMI: body mass index; HR: heart rate; SBP: systolic blood pressure; DBP: diastolic blood pressure; $\mathrm{VO}_{2 \text { peak: }}$ : peak oxygen consumption; daily insulin: long action insulin. 
A



B

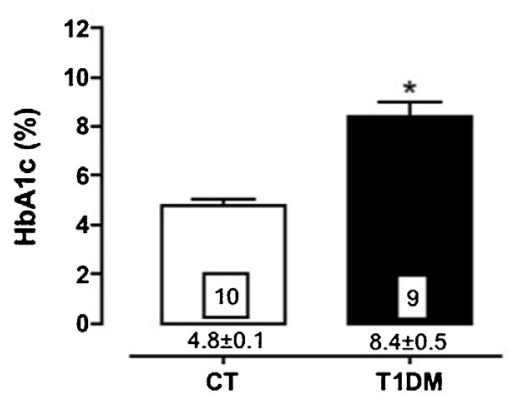

C

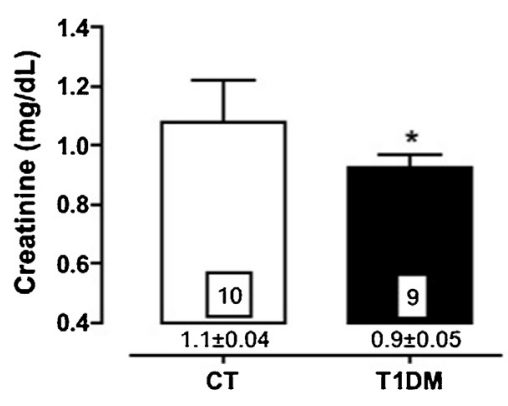

Fig. 2 - Blood glucose, glycated hemoglobin and creatinine. Blood glucose (panel A), glycated hemoglobin (HbA1c, panel B), creatinine (panel C) from control (CT) and type 1 diabetes groups (T1DM). Data are mean \pm SEM. The number of subjects is indicated in the figure. ${ }^{*} p<0.05$ compared with CT.

glucose reduction was greater in T1DM group compared with the CT group (Fig. 4B) $(p<0.001)$.

Concentrations of lactate did not differ between the groups at baseline (Fig. 4C). When we examined the behavior of lactate concentration by delta determination, at 10,20, and 30 min after starting exercise, we found a similar increment in both groups (Fig. 4D) $(p<0.001)$.

\subsubsection{Heart rate and heart rate recovery}

Although baseline heart rate values were not different between the two groups, during the challenge of a bout of exercise, as well as $10 \mathrm{~min}$ after recovery, a greater increase in heart rate value was observed in the T1DM group compared with the CT group (Fig. 5A) $(p<0.05)$. Nevertheless, it should be stressed that both groups performed the aerobic exercise in the target zone of moderate intensity $\left(40-60 \% \mathrm{HR}_{\text {reserve }}\right)$. As heart rate recovery is considered a marker of decreased vagal activity after exercise testing, this was also measured. No differences were found in the values of HR recovery between the groups (CT $=30 \pm 4$ and $\mathrm{T} 1 \mathrm{DM}=28 \pm 4.7 \mathrm{bpm}$ ).

\subsubsection{Heart rate variability}

Considering the relevance of autonomic balance in people with diabetes as well as the importance of heart rate variability for the risk of cardiovascular diseases, we examined heart rate variability in the T1DM group on insulin therapy using time and frequency domain analyses before, during, and after an aerobic exercise session. We found that mean RR and SDNN were reduced at the basal level as well as during the recovery time (Fig. 5, panels B and C) $(p<0.05$ and $p<0.001$, respectively). RMSSD and pNN50 were also significantly reduced in the T1DM group compared with the CT group during the recovery time, but not at baseline (Fig. 5, panels $\mathrm{D}$ and $\mathrm{E})(p<0.01)$.

Interestingly, when we examined the frequency domains, no changes were observed in the LF and HF components of heart rate variability as well as in the LF/HF ratio during all studied period for the two groups (Fig. 5, panels F, G, and $\mathrm{H})$.

\section{Discussion}

The main findings of this study are that people with T1DM without overt cardiovascular diseases presented lower HRV, measured by time domain components, associated with a marked increase in TBARS and a decrease in $\mathrm{NO}_{x}{ }^{-}$concentrations at baseline. Moreover, the alteration in HRV was clearly demonstrated after a bout of aerobic exercise by a slow return to basal values of RMSSD during the recovery period and no return in PNN50, indicating an impairment of parasympathetic tone in this particular population.

\subsection{Baseline measurements}

Studies of diabetes largely focus on oxidative stress and cardiovascular diseases, mainly in T2DM [5]; however, few studies have examined people with T1DM. Our study shows that people with T1DM have poor glucose control, even on insulin therapy. Indeed, previous studies have shown that the efficacy of insulin glargine is poorer at controlling glycemia in people with T1DM [19]. People with diabetes should have a healthy-eating plan contributing to better blood glucose control. Thus, persistent hyperglycemia causes an activation of complex signaling pathways including the protein kinase $C$ pathway, polyol pathway, and AGE formation [3], leading to massive ROS production as a consequence of an imbalance in redox state and oxidative stress. Interestingly, we found no alterations in CML concentration, an AGE biomarker, in the T1DM group compared with the age-matched control group. In contrast, other studies have systemically shown an increased AGE concentration in young adults [20] and middle-aged $[21,22]$ people with T1DM that was independent of the duration of the T1DM. The absence of alterations in CML could be the involvement of another signaling pathway in this particular population, since multiple complex signaling pathways are activated in diabetes states. On the other hand, our 
A

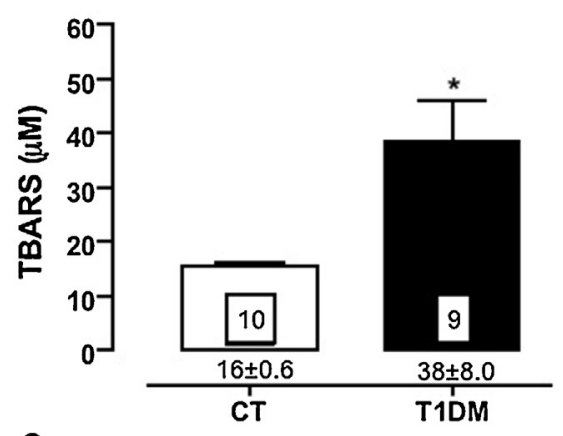

C

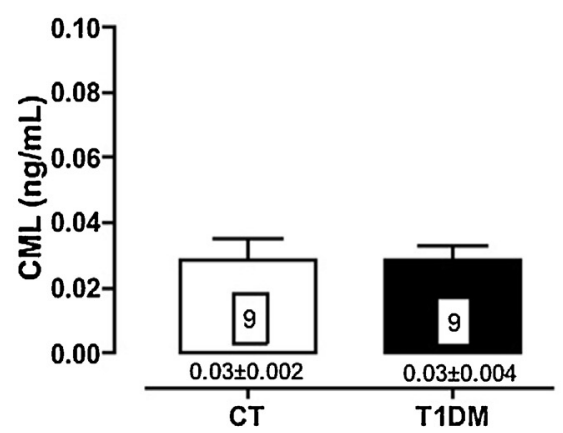

B

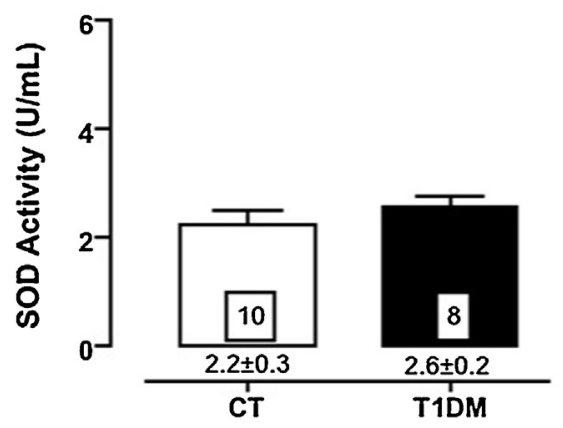

D



Fig. 3 - Plasma biomarkers of oxidative stress, $\mathbf{N}^{\varepsilon}$-(carboxymethyl) lysine and nitrite/nitrate. Plasma thiobarbituric acid reactive substances (TBARS, panel A), superoxide dismutase activity (SOD, panel B), $\mathbf{N}^{\varepsilon}$-(carboxymethyl) lysine (CML, panel C) and nitrite/nitrate ( $\mathrm{NO}_{x}{ }^{-}$, panel D) from control (CT) and type 1 diabetes groups (T1DM). Data are mean \pm SEM. The number of subjects is indicated in the figure. ${ }^{*} p<0.05$ compared with CT. For SOD activity one sample from T1DM group was not detected; For CML one sample from CT group was not detected; $\mathrm{For}_{\mathrm{NO}_{\mathrm{x}}}{ }^{-}$two samples from CT group and one sample from T1DM group were not detected.
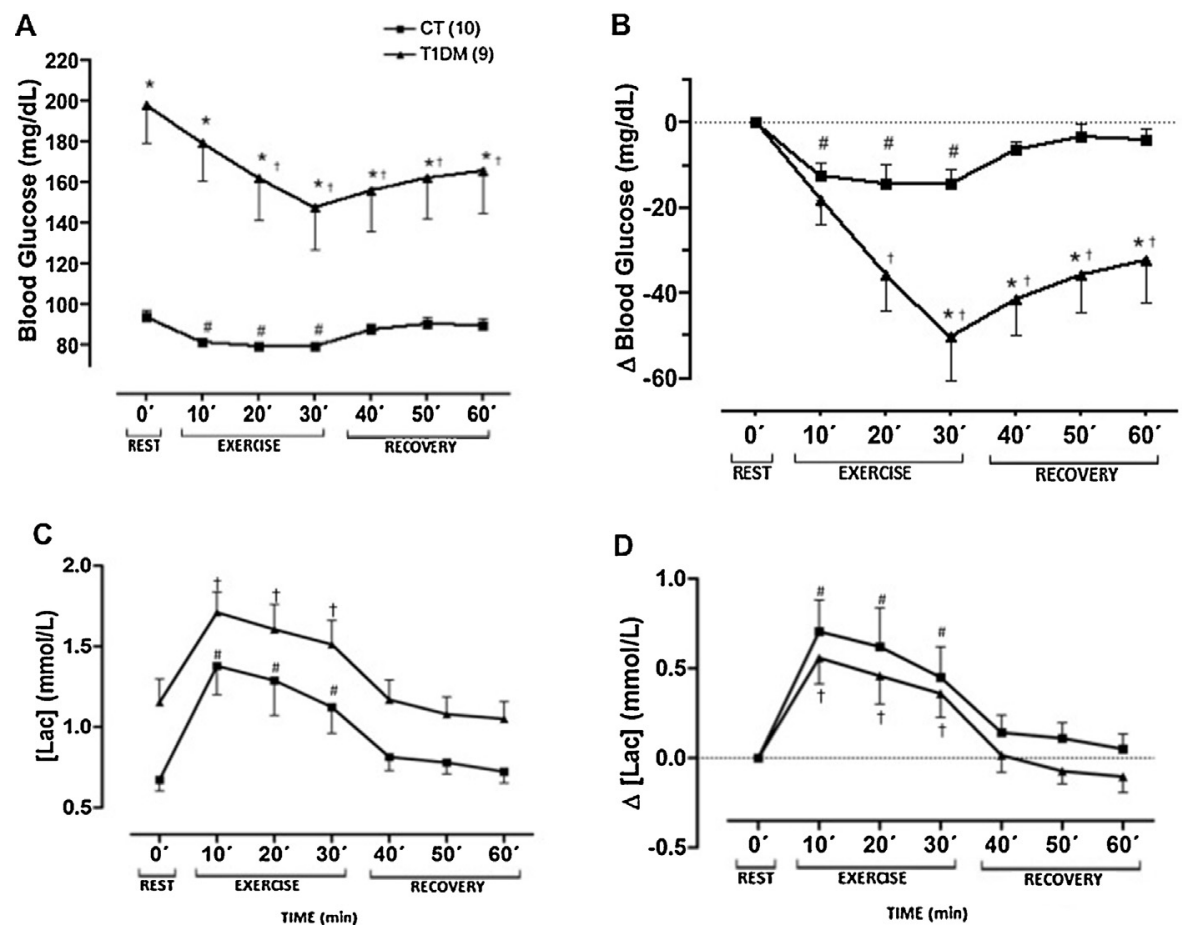

Fig. 4 - Blood glucose and lactate concentration during acute exercise. Blood glucose (panel A), delta reduction of blood glucose (panel B), lactate concentration (panel C), delta reduction of lactate concentration (panel D) from control (CT) and type 1 diabetes groups (T1DM). Data are mean \pm SEM. The number of subjects is indicated in the figure. ${ }^{*} p<0.05$ compared with CT; ${ }^{\#} p<0.05$ compared with CT-T0'; ' $p<0.05$ compared with T1DM-TO'. 
A

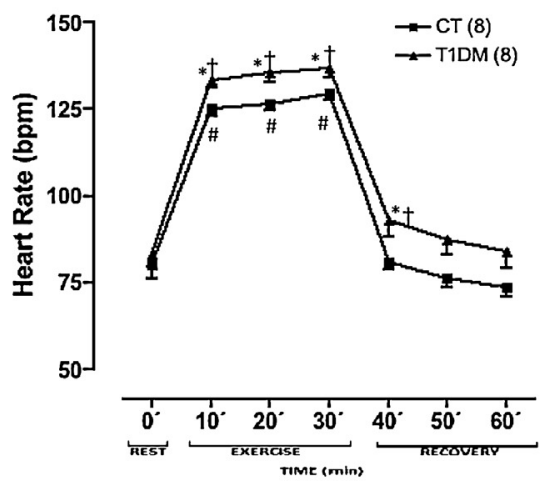

B



C

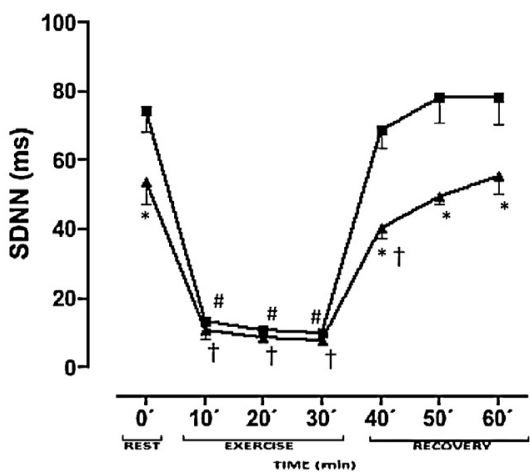

F

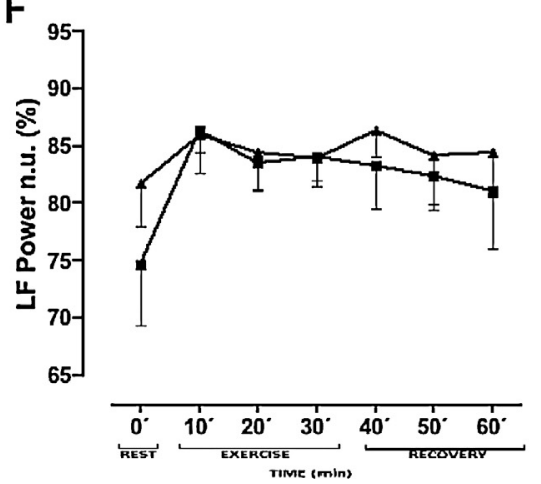

D

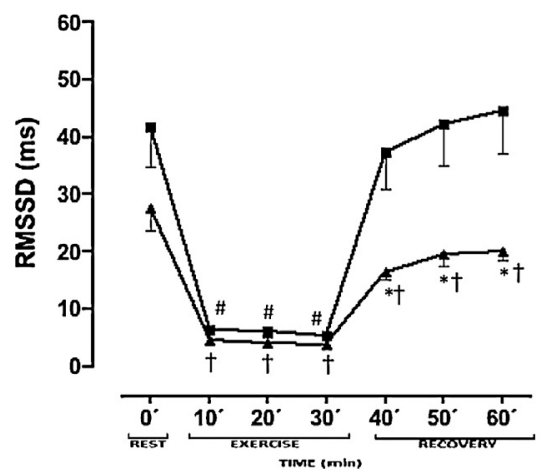

G



E
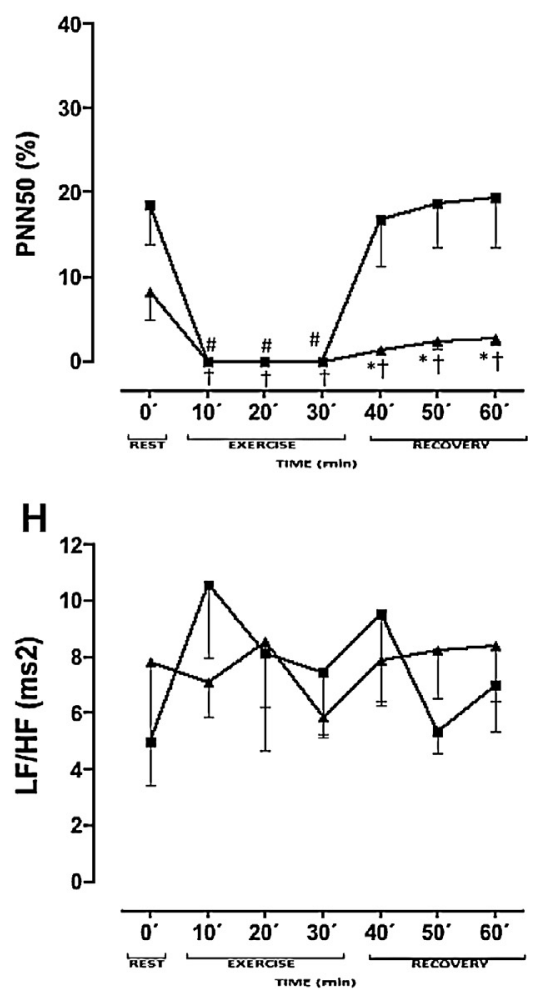

Fig. 5 - Heart rate variability. Heart rate (panel A), mean R-R interval (time between normal cardiac cycles, panel B), the standard deviation of normal R-R intervals (SDNN, panel C), root mean square of successive R-R interval differences (RMSSD, panel D), the proportion of normal R-R intervals exceeds $50 \mathrm{~ms}$ divided by total number of normal R-Rs (pNN50, panel E), low frequency power component (LF power, panel F), high frequency power component (HF power, panel G) and low frequency/high frequency ratio (LH/HF, panel H). Data are mean $\pm \mathrm{SEM}$. The number of subjects is indicated in the figure. ${ }^{*} p<0.05$ compared with CT; ${ }^{*} p<0.05$ compared with CT-T0'; ${ }^{\dagger} p<0.05$ compared with T1DM-TO'. Two subjects from CT group and one subject from T1DM were excluded because the heart beat resulted in errors in the calculation of HRV.

findings clearly show a marked increase in lipid peroxidation biomarkers (140\%) as well as a decrease in $\mathrm{NO}_{x}{ }^{-}$concentration that indirectly reflects NO production or its bioavailability. Accordingly, previous studies demonstrated an increment in TBARS in T1DM patients [23,24]. Thus, the poor glucose control seen in T1DM seems to deeply affect metabolic regulation even in the absence of overt cardiovascular diseases or kidney dysfunction.

Regarding NO production and T1DM, data are controversial and few studies exist examining this biomarker. Increased NO concentrations have been reported $[25,26]$ whereas other studies have failed to detect any changes in endothelium mediators [27], nitrite levels [28], or $\mathrm{NO}_{x}{ }^{-}[24,29]$ in T1DM patients. Differently to other findings, our data show lower concentrations of $\mathrm{NO}_{x}^{-}(60 \%)$ in the $\mathrm{T} 1 \mathrm{DM}$ population suggesting impaired vascular function. The reason for this discrepancy could be because the studied population was young adults without overt cardiovascular diseases. Regarding antioxidant enzyme, our findings show no changes in SOD activity in the T1DM group. Indeed, previous studies failed to 
demonstrate any changes in this important oxidant enzymatic defense $[23,24,30]$.

A number of studies have shown an autonomic imbalance in people with diabetes with alterations in time domain components, with lower SDNN and PNN50 [31,32], as well as lower oscillations in HF components that reflect parasympathetic tone, and a marked prevalence in sympathetic tone, evaluated by LF components $[10,12,33]$. In accordance with previous studies, we found a significant reduction in time domain components (means RR and SDNN) at baseline, but no changes in LF or HF components.

\subsection{Acute aerobic exercise session: heart rate variability and heart rate recovery}

In order to examine the effects of an acute aerobic exercise session and recovery period on HRV, the T1DM group underwent a 30 min treadmill session at an intensity of 40 $60 \% \mathrm{HR}_{\text {reserve. }}$ As expected, during exercise the time domain components were similar between the T1DM group and the age-matched control group. Interestingly, SDNN, a marker of overall HRV, returns promptly to basal values during the recovery time (approximately $7 \mathrm{~min}$ ) in the control group, whereas in the T1DM group, SDNN only returned to baseline values after $30 \mathrm{~min}$. Moreover, RMSSD and PNN50, which reflect the integrity of parasympathetic tone, did not return to basal values during the total 30 min recovery period. Indeed, PNN50 was below 20\%, which is related to high risk of development of cardiac disease. Collectively, our data clearly show an imbalance in autonomic nervous system regulation in T1DM, mainly related to the integrity of vagus nerve to the heart. Intriguing, the behavior of the frequency components was similar between the two groups in response to the exercise session and during the recovery period with increments of LF and decreases in HF components. Regarding heart rate recovery, we found no differences between the groups. The reason for that could be due to the age of the T1DM group as well as absence of overt cardiovascular disease. Indeed, previous studies have shown a positive relationship between lower heart rate recovery and cardiovascular mortality [34,35]. However, the age of the populations of those studies was greater than 55 years, i.e., mainly middle-aged or elderly patients.

In conclusion, our study shows that people with T1DM on insulin therapy have an imbalance in autonomic function, mainly related to parasympathetic tone, which can be detected during the recovery period after a session of aerobic exercise. This alteration is positively associated with poor glycemic control with a greater lipid peroxidation and lower $\mathrm{NO}_{x}{ }^{-}$levels, suggesting a higher risk of developing vascular dysfunction and cardiac disease. The importance of these data highlights the necessity of screening T1DM patients with different tests during clinical practice in an attempt to prevent cardiovascular complications and autonomic neuropathy.

\subsection{Study limitations}

One limitation of the present study was the number of participants. Using a larger study population could lead to new clinical perspectives for prevention of autonomic dysfunction in T1DM. Additionally, in this study only males were evaluated and it would be interesting to compare gender differences in this relevant cardiometabolic disease as well as the pathophysiological events.

\section{Conflict of interest statement}

The authors declare that there are no conflicts of interest.

\section{Funding}

Grants from Sao Paulo Research Foundation to Angelina Zanesco (Number: 2011/17437-7) and Maria A Delbin (Number: 2013/02960-1).

\section{R E F E R E N C E S}

[1] Maritim AC, Sanders RA, Watkins 3rd JB. Diabetes, oxidative stress, and antioxidants: a review. J Biochem Mol Toxicol 2003;17(1):24-38.

[2] Schalkwijk CG, Stehouwer CD. Vascular complications in diabetes mellitus: the role of endothelial dysfunction. Clin Sci 2005;109(2):143-59.

[3] Goldin A, Beckman JA, Schmidt AM, Creager MA. Advanced glycation end products: sparking the development of diabetic vascular injury. Circulation 2006;114(6):597-605.

[4] Frey RS, Ushio-Fukai M, Malik AB. NADPH oxidase-dependent signaling in endothelial cells: role in physiology and pathophysiology. Antioxid Redox Signal 2009;11(4):791-810.

[5] Forbes JM, Cooper ME. Mechanisms of diabetic complications. Physiol Rev 2013;93(1):137-88.

[6] Libby P, Nathan DM, Abraham K, Brunzell JD, Fradkin JE, Haffner SM, et al. National Heart, Lung, and Blood Institute; National Institute of Diabetes and Digestive and Kidney Diseases Working Group on Cardiovascular Complications of Type 1 Diabetes Mellitus. Report of the National Heart, Lung, and Blood Institute-National Institute of Diabetes and Digestive and Kidney Diseases Working Group on Cardiovascular Complications of Type 1 Diabetes Mellitus. Circulation 2005;111(25):3489-93.

[7] Vinik AI, Erbas T. Diabetic autonomic neuropathy. Handb Clin Neurol 2013;117:279-94

[8] Heart rate variability: standards of measurement, physiological interpretation, and clinical use. Task Force of the European Society of Cardiology and the North American Society of Pacing and Electrophysiology. Eur Heart J 1996;17(3):354-81.

[9] Billman GE, Huikuri HV, Sacha J, Trimmel K. An introduction to heart rate variability: methodological considerations and clinical applications. Front Physiol 2015;6:55

[10] Bernardi L, Ricordi L, Lazzari P, Soldá P, Calciati A, Ferrari MR, et al. Impaired circadian modulation of sympathovagal activity in diabetes. A possible explanation for altered temporal onset of cardiovascular disease. Circulation 1992;86(5):1443-52.

[11] Vinik AI, Maser RE, Mitchell BD, Freeman R. Diabetic autonomic neuropathy. Diabetes Care 2003;26(5):1553-79.

[12] Tannus LR, Sperandei S, Montenegro Júnior RM, Carvalho VR, Pedrosa HC, Félix MT, et al. Reproducibility of methods used for the assessment of autonomous nervous system's function. Auton Neurosci 2013;177(2):275-9. 
[13] American Diabetes Association. Standards of medical care for patients with diabetes mellitus. Diabetes Care 1997;20:S3-13.

[14] Fiuza-Luces C, Garatachea N, Berger NA, Lucia A. Exercise is the real polypill. Physiology 2013;28(5):330-58.

[15] International Diabetes Federation Diabetes Atlas. 6th ed., 2013.

[16] Widrick J, Ward A, Ebbeling C, Clemente E, Rippe JM. Treadmill validation of an over-ground walking test to predict peak oxygen consumption. Eur J Appl Physiol Occup Physiol 1992;64(4):304-8.

[17] Karvonen MJ, Kentala E, Mustala O. The effects of training on heart rate; a longitudinal study. Ann Med Exp Biol Fenn 1957;35(3):307-15.

[18] Eengels RC, Jones JB. Causes and elimination of erratic blanc in enzymatic metabolic assays involving the use of NAD in alkaline hydrazine buffers: improved conditions for assay of L-glutamate, L-lactate and other metabolites. Anal Biochem 1978;88:475-84.

[19] Rosenstock J, Bergenstal RM, Blevins TC, Morrow LA, Prince $\mathrm{MJ}, \mathrm{Qu} \mathrm{Y}$, et al. Better glycemic control and weight loss with the novel long-acting basal insulin LY2605541 compared with insulin glargine in type 1 diabetes: a randomized, crossover study. Diabetes Care 2013;36(3):522-8.

[20] Galler A, Müller G, Schinzel R, Kratzsch J, Kiess W, Münch G. Impact of metabolic control and serum lipids on the concentration of advanced glycation end products in the serum of children and adolescents with type 1 diabetes, as determined by fluorescence spectroscopy and nepsilon(carboxymethyl)lysine ELISA. Diabetes Care 2003;26(9):2609-15.

[21] Lieuw-A-Fa ML, van Hinsbergh VW, Teerlink T, Barto R, Twisk J, Stehouwer CD, et al. Increased levels of N(epsilon)(carboxymethyl)lysine and N(epsilon)-(carboxyethyl)lysine in type 1 diabetic patients with impaired renal function: correlation with markers of endothelial dysfunction. Nephrol Dial Transplant 2004;19(3):631-6.

[22] Nin JW, Jorsal A, Ferreira I, Schalkwijk CG, Prins MH, Parving $\mathrm{HH}$, et al. Higher plasma levels of advanced glycation end products are associated with incident cardiovascular disease and all-cause mortality in type 1 diabetes: a 12-year follow-up study. Diabetes Care 2011;34(2):442-7.

[23] Ruiz C, Alegría A, Barberá R, Farré R, Lagarda MJ. Lipid peroxidation and antioxidant enzyme activities in patients with type 1 diabetes mellitus. Scand J Clin Lab Invest 1999;59(2):99-105. Apr.
[24] Fayh AP, Krause M, Rodrigues-Krause J, Ribeiro JL, Ribeiro JP, Friedman R, et al. Effects of L-arginine supplementation on blood flow, oxidative stress status and exercise responses in young adults with uncomplicated type I diabetes. Eur J Nutr 2013;52(3):975-83.

[25] Mylona-Karayanni C, Gourgiotis D, Bossios A, Kamper EF. Oxidative stress and adhesion molecules in children with type 1 diabetes mellitus: a possible link. Pediatr Diabetes 2006;7(1):51-9. Feb.

[26] Horoz OO, Yuksel B, Bayazit AK, Attila G, Sertdemir Y, Mungan NO, et al. Ambulatory blood pressure monitoring and serum nitric oxide concentration in type 1 diabetic children. Endocr J 2009;56(3):477-85.

[27] Smulders RA, Stehouwer CD, Olthof CG, van Kamp GJ, Teerlink T, de Vries PM, et al. Plasma endothelin levels and vascular effects of intravenous L-arginine infusion in subjects with uncomplicated insulin-dependent diabetes mellitus. Clin Sci 1994;87(1):37-43.

[28] Ferlito S, Gallina M. Nitrite plasma levels in type 1 and 2 diabetics with and without complications. Minerva Endocrinol 1999;24(3-4):117-21.

[29] Farkas K, Jermendy G, Herold M, Ruzicska E, Sasvári M, Somogyi A. Impairment of the NO/cGMP pathway in the fasting and postprandial state in type 1 diabetes mellitus. Exp Clin Endocrinol Diabetes 2004;112(5):258-63.

[30] Woo J, Yeo NH, Shin KO, Lee HJ, Yoo J, Kang S. Antioxidant enzyme activities and DNA damage in children with type 1 diabetes mellitus after 12 weeks of exercise. Acta Paediatr 2010;99(8):1263-8.

[31] Ewing DJ, Neilson JM, Travis P. New method for assessing cardiac parasympathetic activity using 24 hour electrocardiograms. Br Heart J 1984;52(4):396-402.

[32] Turker Y, Aslantas Y, Aydin Y, Demirin H, Kutlucan A, Tibilli $\mathrm{H}$, et al. Heart rate variability and heart rate recovery in patients with type 1 diabetes mellitus. Acta Cardiol 2013;68(2):145-50.

[33] Jaiswal M, Fingerlin TE, Urbina EM, Wadwa RP, Talton JW, D'Agostino Jr RB, et al. Impact of glycemic control on heart rate variability in youth with type 1 diabetes: the SEARCH CVD study. Diabetes Technol Ther 2013;15(12):977-83.

[34] Cole CR, Blackstone EH, Pashkow FJ, Snader CE, Lauer MS. Heart-rate recovery immediately after exercise as a predictor of mortality. N Engl J Med 1999;341(18):1351-7.

[35] Myers J, Tan SY, Abella J, Aleti V, Froelicher VF. Comparison of the chronotropic response to exercise and heart rate recovery in predicting cardiovascular mortality. Eur J Cardiovasc Prev Rehabil 2007;14(2):215-21. 